\title{
Mechatronic Systems Design for an Autonomous Robotic System for High-Efficiency Bridge Deck Inspection and Evaluation
}

\author{
Hung M. La, Ronny Salim Lim, Basily B. Basily, Nenad Gucunski, Jingang Yi, Senior Member, IEEE, \\ Ali Maher, Francisco A. Romero, and Hooman Parvardeh
}

\begin{abstract}
The condition of bridges is critical for the safety of the traveling public. Bridges deteriorate with time as a result of material aging, excessive loading, environmental effects, and inadequate maintenance. The current practice of nondestructive evaluation (NDE) of bridge decks cannot meet the increasing demands for highly efficient, cost-effective, and safety-guaranteed inspection and evaluation. In this paper, a mechatronic systems design for an autonomous robotic system for highly efficient bridge deck inspection and evaluation is presented. An autonomous holonomic mobile robot is used as a platform to carry various NDE sensing systems for simultaneous and fast data collection. The robot's NDE sensor suite includes ground penetrating radar arrays, acoustic/seismic arrays, electrical resistivity sensors, and video cameras. Besides the NDE sensors, the robot is also equipped with various onboard navigation sensors such as global positioning system (GPS), inertial measurement units (IMU), laser scanner, etc. An integration scheme is presented to fuse the measurements from the GPS, the IMU and the wheel encoders for high-accuracy robot localization. The performance of the robotic NDE system development is demonstrated through extensive testing experiments and field deployments.
\end{abstract}

Index Terms-Bridge deck evaluation, civil infrastructure, mobile robot, nondestructive evaluation (NDE), sensor fusion.

\section{INTRODUCTION}

B RIDGES are one of the infrastructure components critical for the safety of the traveling public and the sustainability

Manuscript received December 31, 2012; revised April 11, 2013; accepted August 17, 2013. Date of publication September 16, 2013; date of current version December 11, 2013. Recommended by Guest Editor Y. Wang. This paper was presented in part at the 2013 IEEE International Conference on Automation Science and Engineering, Madison, WI, USA, August 18-20. This work was supported in part by the U.S. National Institute of Standards and Technology (NIST) Technology Innovation Program (TIP) under Award 70NANB10H014. (Corresponding author: J. Yi.)

H. M. La, R. S. Lim, and H. Parvardeh are with the Center for Advanced Infrastructure and Transportation, Rutgers University, Piscataway, NJ 08854 USA (e-mail: hung.la11@ rutgers.edu; ronny.lim@ rutgers.edu; hoomanp@ rci. rutgers.edu).

B. B. Basily is with the Department of Industrial and Systems Engineering, Rutgers University, Piscataway, NJ 08854 USA (e-mail: basily@ rci.rutgers. edu).

N. Gucunski and A. Maher are with the Department of Civil and Environmental Engineering, Rutgers University, Piscataway, NJ 08854 USA (e-mail: gucunski@ rci.rutgers.edu; mmaher@ rci.rutgers.edu).

$\mathrm{J}$. Yi is with the Department of Mechanical and Aerospace Engineering, Rutgers University, Piscataway, NJ 08854 USA (e-mail: jgyi@ rutgers.edu).

F. A. Romero was with the Center for Advanced Infrastructure and Transportation, Rutgers University, Piscataway, NJ 08854 USA . He is now with the GPR TEAM Board of Directors (e-mail: faromero@ @rci.rutgers.edu).

Color versions of one or more of the figures in this paper are available online at http://ieeexplore.ieee.org.

Digital Object Identifier 10.1109/TMECH.2013.2279751

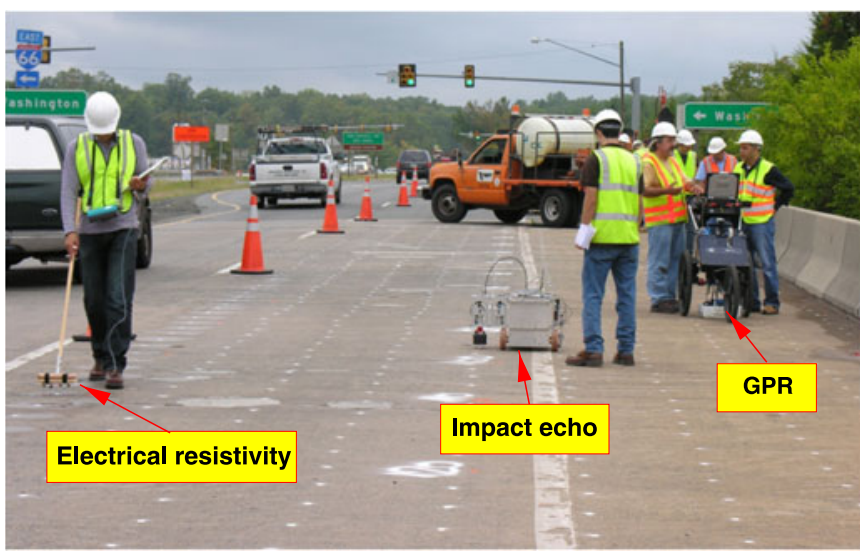

Fig. 1. State-of-the-art of the NDE technology for bridge deck inspection and evaluation.

of the economic activity. In the United States, there are currently more than 600000 bridges. They deteriorate with time as a result of material aging, excessive use, and overloading, environmental conditions, inadequate maintenance, and deficiencies in inspection and evaluation. According to the American Association of State Highway and Transportation Officials (AASHTO) and the U.S. Federal Highway Administration (FHWA), the cost of repairing and replacing deteriorating highway bridges in U.S. only is estimated at more than $\$ 140$ billions in 2008 [1], [2]. The potential social impact of these deteriorating infrastructure to the general public is enormous.

Effective health monitoring, maintenance, repair, rehabilitation, and the replacement of the deteriorating bridge components are necessary to ensure the transportation safety [3]. Nondestructive evaluation (NDE) is one of the effective ways to reliably identify and predict early-stage bridge deterioration to enable proactive interventions for repair and rehabilitation. There are a number of NDE technologies that are currently being used in bridge evaluation, including impact echo, ground penetrating radar (GPR), electrical resistivity, visual inspection, etc. [4], [5]. Fig. 1 illustrates the application of NDE technologies in bridge deck inspection. As shown in the figure, when NDE is conducted on bridge decks, a section of the bridge is closed for traffic causing inconvenient traffic interruptions. The delivery of the current NDE technologies cannot meet the increasing demands for highly efficient, cost-effective, and safety-guaranteed inspection and evaluation in several aspects. First, since each NDE technique is conducted manually, the data collection 


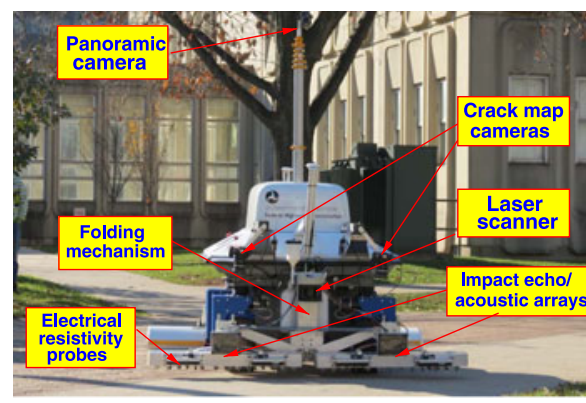

(a)

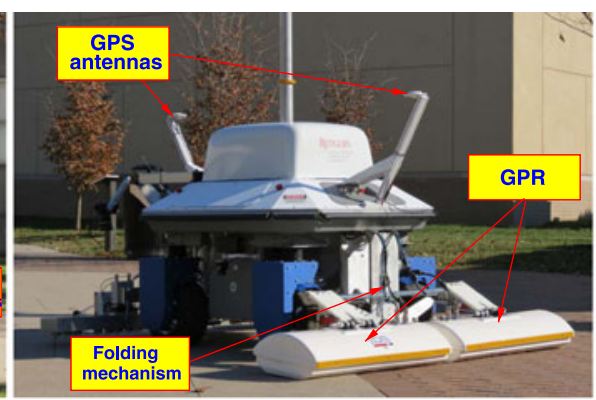

(b)

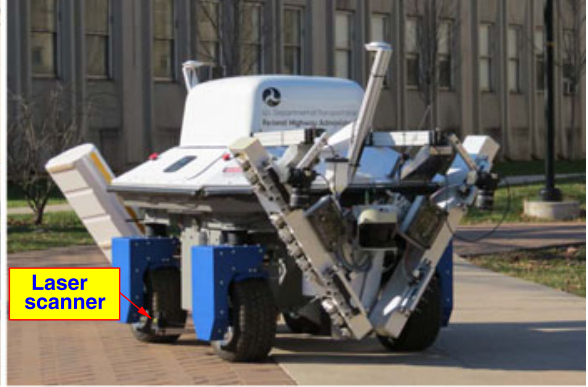

(c)

Fig. 2. Rutgers robotic bridge deck diagnosis system. (a) Front view of the system while the NDE sensors are at working position. (b) Rear view of the robotic system. (c) Side view of the robotic system when the NDE sensors are at idle position.

is prone to human error. Moreover, there are safety risks for inspectors as vehicles run in the adjacent lanes. Second, the capacity of the manual NDE inspection is highly limited due to the speed of data collection. Third, the cost of the manual inspection is high because it is labor intensive work and has negative impacts on the traffic flow. Finally, in most cases only individual NDE technologies are deployed instead of the use of multiple complementary NDE technologies that can provide comprehensive evaluation.

The goal of this paper is to present the development and demonstration of a mechatronic systems design for a new autonomous robotic system for high-efficiency bridge deck inspection and evaluation. Robotics and automation technologies have gained increasingly applications in civil infrastructure in the past two decades. For example, mobile robot- or vehiclebased inspection systems were developed for cracks detection and maintenance for highways [6]-[9], and tunnels [10], [11]. The mobile manipulator systems were used to extend the capability of human inspectors for bridge crack inspection [12] and rehabilitation [13], [14]. Similar systems were developed for vision-based automatic crack detection and mapping [15]-[17] and paint removal [18] for bridges. A set of mobile robots with magnetic wall-climbing capability were developed to enable a wireless sensor network for steel bridge condition monitoring [19]. Despite all of the aforementioned developments, to the best of the author's knowledge, no autonomous robotic system is developed to deploy and integrate multiple NDE technologies for high-efficiency and high-accuracy bridge deck condition assessment.

One of the main challenging tasks in the development of an autonomous robot for bridge deck inspection is a robust, reliable robotic localization and navigation system. Since the robotic system needs to cover the narrow deck surface, it is required that the localization and navigation accuracy be within a range of a few centimeters. Although high-accuracy global positioning system (GPS) with a real-time kinematic (RTK) correction can reach the requirement, it is well known that GPS signals are not always reliable and robust, especially on bridges with partial coverage, steel cables, truss elements, or other support structures. Similar to the approaches in [20]-[22], dual RTK GPS antennas are used on the developed mobile robot platform. The GPS measurements are integrated with attitude information from the onboard inertial measurement unit (IMU) to enhance the localization accuracy. Moreover, the developed navigation system also fuses the GPS/IMU measurements with the wheel odometry information through an extended Kalman filter (EKF) design [23]-[25]. The accuracy of the wheel odometry of the all-wheel steering platform is much higher than those of other types of mobile robots (e.g., car-like or skid-steered mobile robots) due to the small wheel slippages in operation [24], [26]. With the odometry-enhanced GPS/IMU navigation, the robotic system has demonstrated high-accuracy localization that meets the inspection requirements even in GPS-denied environments.

The main contributions of this study are twofold. First, a new autonomous robotic system is presented for highly efficient NDE for bridge decks. The robotic system is the first of this kind of autonomous systems that integrates the state-of-theart robotics and NDE technologies. Second, the performance of the robotic system enables fast data collection and highly accurate bridge deck inspection and evaluation. In this process, the robotic system utilizes data fusion of multiple NDE technologies for the overall condition assessment. Compared to the state-ofthe-art NDE technologies, the use of the developed autonomous robotic bridge deck inspection system will significantly reduce the cost and time of inspection. This work is a significant extension of the previously presented conference publication [27].

The rest of this paper is organized as follows. In Sections II and III, an overview of the robotic system design is presented, including a description of the NDE sensors and the robotic integration. In Section IV, the robot localization and navigation systems are presented. The samples of the robotic navigation experiments and results are presented in Section V. Finally, the conclusions and discussion of future work are presented in Section VI.

\section{OVERVIEW OF THE ROBOTIC SYSTEM}

The robotic system with integrated NDE technologies is shown in Fig. 2. The mobile platform is a Seekur robot from Adept Mobile Robot Inc. The Seekur robot is an electrical allwheel driving and steering platform. Such a holonomic mobile robot was chosen primarily because of the required high-agility motions on narrow bridge decks, such as zero-turning-radius maneuver and parallel movement, etc. The Seekur robot is also an all-weather outdoor platform that is desirable for the bridge inspection applications. The control hierarchy for the mobile 


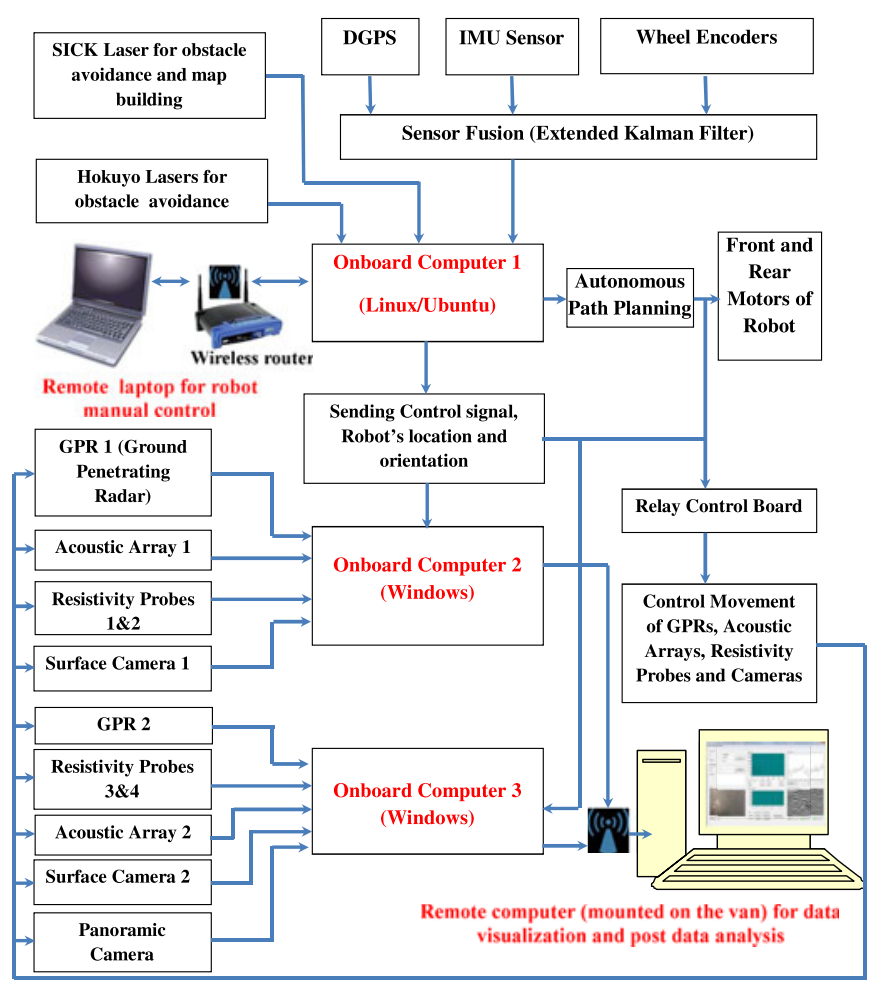

Fig. 3. Architecture of the mobile robot system for bridge deck evaluation and inspection.

robot consists of two layers: the lower-level real-time robot controllers are provided by the vendor and the upper level control is supplied by the users. The upper-level controller provides the desired linear velocity and the yaw angle values for the robot, while the lower-level controller takes these commanded values and drives the motors for desired robot motion.

The mobile robot has been modified and equipped with various sensors, actuators, and computing devices. The robot is equipped with two sets of sensor suites: navigation and motion planning sensors and NDE sensors. The navigation and motion planning sensors include two RTK GPS units (from Novatel, Inc.), one front- and two side-mounted laser scanners (from Sick AG and Hokuyo Automation Co., respectively), and one IMU sensor (from Microstrain, Inc.) The onboard NDE sensors include two GPR units, two seismic/acoustic array sensors, four electrical resistivity probes, two high-resolution digital cameras and a $360^{\circ}$ panoramic camera. A description of these NDE sensors and their integration is presented in Section III.

The control and computing architecture of the robotic system is shown in Fig. 3. Three industrial standard embedded computers (from Versalogic, Inc.) are installed inside the robot. These computers can operate functionally in high temperature environment (up to $80^{\circ} \mathrm{C}$ ) for all-season field testing. One computer runs Robotic Operating System (ROS) [28] for the robot navigation and motion planning tasks. The other two computers use Windows operating system for integrating the NDE sensors and data collection. High-speed Ethernet connections are used among these computers and each computer can also be reached individually through high-speed wireless communication by the remote computers. The NDE data and images are also transmitted in real time to the remote computers for visualization and data analysis purposes. The remote visualization and data analysis computers are located inside a full-size cargo van that is also used to transport the robotic system.

Some NDE sensors including seismic/acoustic sensors and electrical resistivity probes need to be in contact with the deck surface for the measurement. Therefore, a set of pneumatic actuators were built to move these NDE sensors up from and down to the deck surface. Typically, the robot moves and stops at a certain distance (e.g., $0.6 \mathrm{~m}$ ) for NDE data collection. When the robot stops, the NDE sensor probes are coupled to the deck surface. When the data collection is finished, the NDE probes are lifted up and the robot is ready to move to the next planned inspection point. The motion coordination between the NDE sensors and the robot navigation is accomplished through the control algorithms.

A remote graphic user interface (GUI) is developed for the robotic system to allow end users to easily operate the robot and the NDE sensors as well as the data visualization. The GUI is developed in the ROS environment and through the GUI, robot operators and field engineers can remotely control the robot in several modes: manual, semiautonomous, and fully autonomous. Additionally, the GUI also displays the robot data for monitoring purpose, such as position, heading, laser scan, image video stream, bumper state, and battery life. All of the robot navigation information and the collected NDE data can be saved in the remote computers for data processing and analysis.

The design of the robotic system faced two main challenging tasks: one was to seamlessly integrate various NDE sensors with the robotic platform and the other to design a reliable, robust robot localization and navigation system to safely maneuver on narrow bridge decks. The following sections describe the development activities related to these two tasks.

\section{RoBOTIC NDE SYSTEMS}

This section briefly discusses NDE technologies used in the robotic system. This is followed by the presentation of the mechatronic design to integrate the NDE sensors with the robotic platform.

\section{A. NDE Technologies for Bridge Deck Inspection}

1) Ground Penetrating Radar (GPR): GPR is a geophysical method that uses radar pulses to image the subsurface and describe the condition. During the survey, electromagnetic waves are transmitted into the deck. When they encounter objects or materials of different dielectric properties, such as rebars or ducts, the waves are reflected and detected by a receiving antenna. GPR can provide a qualitative condition assessment of bridge decks, in terms of detection of apparent or suspected deterioration (e.g., delamination) description of corrosive environment. The GPR usually assesses the condition of concrete bridge decks based on the attenuation of electromagnetic waves on the top rebar level. The GPR is also used as a quality assurance tool for new construction or rehabilitation. The GPR systems for bridge deck applications use antennas in a frequency 
range from 1 to $2.5 \mathrm{GHz}$. A detailed description of the GPR technology can be found in [29]. The developed robotic system utilizes Hi-Bright ground-coupled GPR arrays manufactured by IDS Italy.

2) Impact Echo: The impact echo method is used in the detection of discontinuities and element thickness measurements. It is a seismic resonant method that is primarily used to detect and characterize delamination (horizontal cracking) in bridge decks with respect to the delamination depth, spread, and severity. It can be also used to detect debonding of overlays on bridge decks. The developed robotic system integrates more than a dozen of the impact echo sensors.

3) Ultrasonic Surface Waves (USW): The USW method is used to assess the concrete quality by measuring its elastic modulus. Low modulus is often related to concrete degradation or delamination. However, lower modulus values are also observed in new decks as a result of material variability and concrete placement procedures. Therefore, only periodical assessment of concrete modulus can be used to detect a deterioration process.

4) Electrical Resistivity: Electrical resistivity sensor measures concrete's electrical resistivity, which is a reflection of the corrosive environment of the bridge deck. The presence of water, chlorides, salts, or other contaminants reduces concrete's resistivity, and facilitates corrosive processes in bridge decks. By measuring the electrical resistivity, the corrosion rate of reinforcing rebars can be estimated. To ensure good coupling between the electrodes of the resistivity probe and concrete, water is lightly sprayed on the electrodes.

5) Visual Detection of Surface Cracks: The computer vision is an efficient and effective method to detect and map the surface cracks. However, developing a reliable and robust vision-based crack detection and mapping system is a challenging task due to the variations in the outdoor environment, such as illumination conditions, and ill-structured deck surface images. The developed robotic system uses two front cameras to capture the deck surface images for crack detection/mapping and one panoramic camera with a $360^{\circ}$ field of view to capture the surveyed area. The panoramic camera is mounted on a computer-controlled, extendable mast.

The previously discussed NDE sensing technologies are used in a complementary matter to provide comprehensive information about the bridge deck condition [4], [30].

\section{B. Mechatronic Design for Robotic NDE Sensor Integration}

A set of mechatronic components were designed to integrate the NDE sensors with the mobile robot. The design goal was to deploy the NDE sensors with the mobile movements for highefficiency, fast NDE data collections. With the robotic NDE sensor design, it is desirable to enable a large-area scanning and coverage of a bridge deck during a single robot pass. Meanwhile, installationn and distribution of the NDE sensors on the robot need to be carefully considered due to the limited onboard battery capacity and payload (less than $150 \mathrm{~kg}$ ). The footprint of the robotic system also needs to be kept compact for transportation and storage.

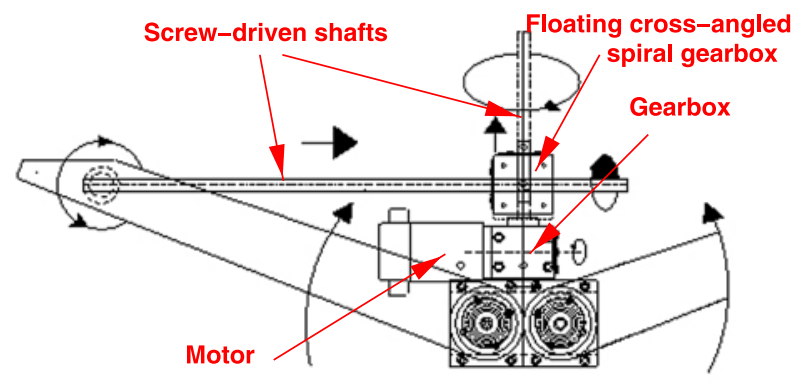

(a)

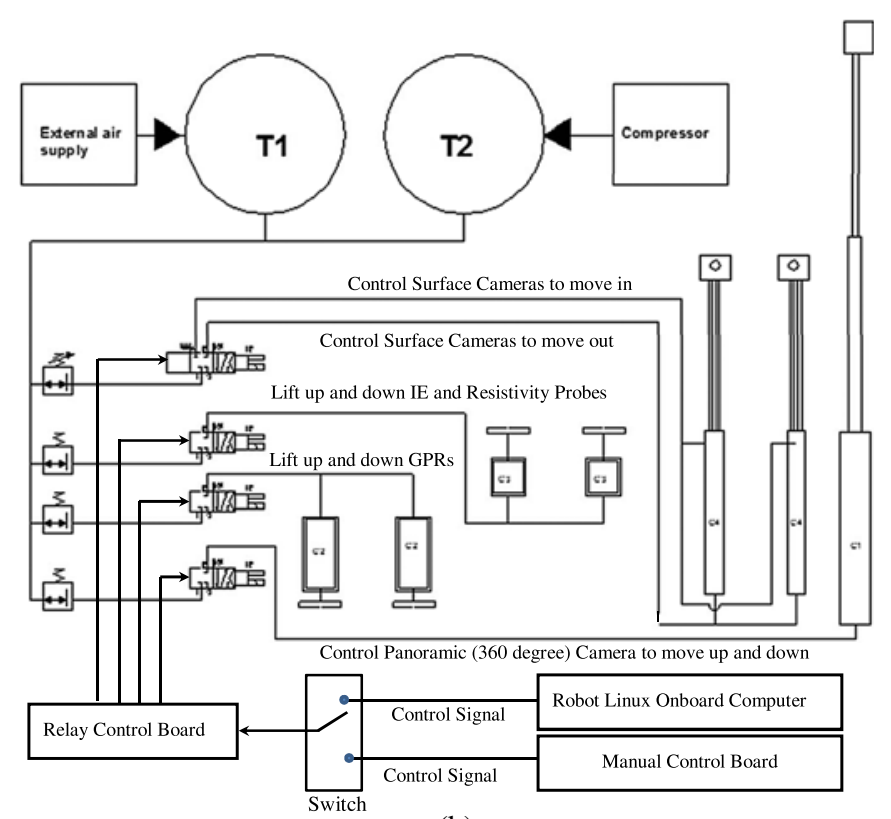

(b)

Fig. 4. (a) Folding mechanism to extend and retreat the GPS antennas, impact echo/acoustic arrays and electrical resistivity probes. (b) Schematic of the pneumatic control circuit for lifting up and laying down NDE sensors and the regulating the cameras positions.

To meet the previous design requirements, an innovative mechanical folding mechanism and a pneumatic actuation design were developed for the robotic NDE sensor integration. Fig. 4(a) illustrates the folding mechanism to extend and retreat the GPR arrays, seismic/acoustic arrays, and electrical resistivity sensor suite to keep a compact design. Fig. 4(b) shows the schematic of the pneumatic actuation design for lifting up and laying down the GPR, acoustic arrays, and electrical resistivity NDE probes to and from the deck surface.

The folding mechanisms are installed on the front (for the seismic/acoustic arrays and electrical resistivity sensors) and the rear (for the GPR antenna arrays) of the robot; see Fig. 2(a) and (b), respectively. Two swing arms lift the sensor units into a folded position through a screw-driven angled spiral gear box, as shown in Fig. 4(a). The floating gearbox is driven by a vertical screwed shaft powered by a high torque geared motor. Folding and unfolding of the sensor units are achieved by simply reversing the motor moving direction, while the arms swing angle is controlled by the end-of-stroke limit switches. Fig. 5(a) and (b) shows the examples of folding and unfolding the 

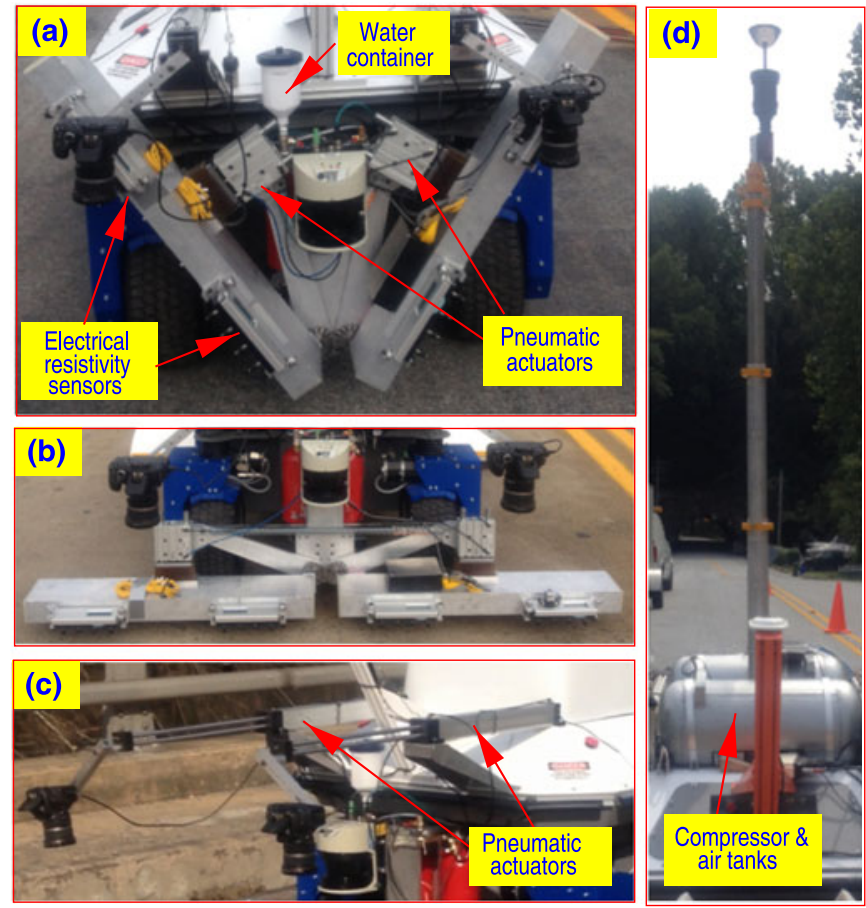

Fig. 5. Snapshots of mechatronic design for the NDE sensor/robot integration. (a) Folding position for the NDE sensors. (b) Unfolding and working position for the NDE sensors. (c) Extended position for the crack detection and mapping cameras. (d) Extended position of telescopic mast for the survey panoramic camera.

seismic/acoustic arrays and the electrical resistivity sensors. This innovative mechanism design provides a stable and highly reliable sensor-support system with minimum energy consumption during the NDE data collection and zero power consumption at the folded end position. For the electrical resistivity probes, an additional water spraying circuit and a pressured water container [see Fig. 5(a)] for wetting of the sensor electrodes are designed and integrated.

A pneumatically expanded telescopic mast is used to lift the panoramic camera up to a height of $4.5 \mathrm{~m}$ above the ground; see Fig. 5(d). The mast's five telescopic segments can collapse the mast to the height matching the top of the robot platform, as shown in Fig. 2. The deployment system for the surface cracks inspection cameras consists of two nonrotating piston rod pneumatic cylinders that are placed $0.8 \mathrm{~m}$ apart; see Fig. 5(c). The pneumatic cylinder has a $0.45 \mathrm{~m}$ stroke which expands to the position where the two cameras lie in the center positions for taking images of a surface area of $2 \times 0.6 \mathrm{~m}^{2}$, including an extra $30 \%$ overlap between the two images that is needed for image stitching. The pneumatic control circuit as shown in Fig. 4(b) includes two light weight compressed air tanks and a portable compressor [see Fig. 5(d)], operating the actuators through pressure regulators and solenoid valves and control unit.

The entire deployment systems are controlled by the robot main computer, or manually through an override switch. The use of the pneumatic circuit provides an additional energy storage capability through external charging of $150 \mathrm{psi}$ compressed air

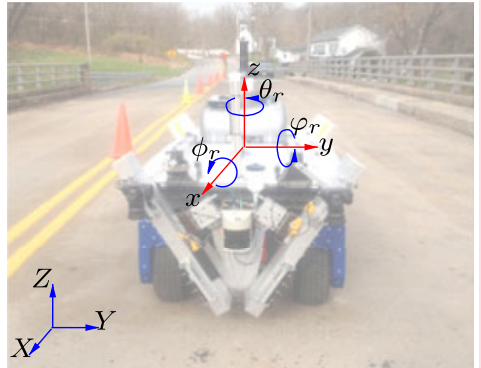

(a)

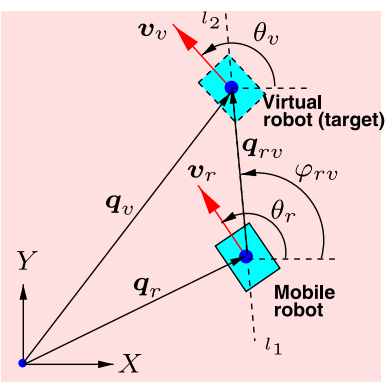

(b)
Fig. 6. (a) Robot attitude coordinates. (b) Schematic of virtual robot tracking design.

for tanks refill. The pneumatic circuit also includes a small low voltage compressor for onboard refill. The manual override unit enables control of the entire deployment system manually in an emergency case of power failure or software crash.

\section{Robot NAVIGATION AND MOTION CONTROL}

A reliable, robust, and highly accurate robot navigation and motion control system was another challenging task in the development of the robotic system. In this section, the development of the robot localization system is discussed. It is followed by the presentation of the motion planning and control scheme to safely maneuver the robot on bridge decks.

\section{A. EKF-Based Robot Localization}

The robotic system is equipped with two RTK GPS units, one IMU unit, and four wheel encoders. Although the RTK GPS unit may possibly provide $2 \mathrm{~cm}$ positioning accuracy under an ideal and clear weather condition, the navigation system cannot rely only on the GPS positioning information for several reasons. First, the GPS measurements are not reliable under allweather conditions. It was observed that under cloudy weather conditions, the accuracy of the RTK GPS data deteriorates significantly. Second, some bridges have been partially covered by steel structures, supporting cables, etc., and under these conditions, the GPS signals are not reliable and robust. To design a reliable, robust localization scheme, the GPS data are fused with the IMU and the wheel encoder measurements through an EKF design.

An inertial frame $\mathcal{I}$ : $X Y Z$ is defined on the bridge deck with the $X$-axis along the traffic flow direction and the $Z$-axis vertically upward, as shown in Fig. 6. The IMU is mounted around the center of the robot and the Euler angles $\boldsymbol{\Phi}=\left[\begin{array}{lll}\phi_{r} & \varphi_{r} & \theta_{r}\end{array}\right]^{T}$ are used to define the robot's attitude, where $\phi_{r}$ is the roll angle, $\varphi_{r}$ the pitch angle and $\theta_{r}$ the yaw angle, as shown in Fig. 6(a). The robot is assumed to be running on a flat bridge deck. The 2-D position vector of the robot center in $\mathcal{I}$ is denoted as $\boldsymbol{q}_{r}=\left[\begin{array}{ll}x_{r} & y_{r}\end{array}\right]^{T}$. Also, the yaw rate of the robot is denoted as $\omega_{\theta}$.

To simplify the localization and motion planning design, the robot's velocity direction is assumed to be aligned with the heading direction and therefore, a unicycle kinematic model is 
used to approximate the robot motion,

$$
\left\{\begin{array}{l}
\dot{x}_{r}=v_{r} \cos \theta_{r}=v_{r} \mathrm{c}_{\theta_{r}} \\
\dot{y}_{r}=v_{r} \sin \theta_{r}=v_{r} \mathrm{~s}_{\theta_{r}}
\end{array}\right.
$$

where $v_{r}$ is the magnitude of the robot linear velocity $\boldsymbol{v}_{r}$ and notations $c_{\theta_{r}}:=\cos \theta_{r}$ and $\mathrm{s}_{\theta_{r}}:=\sin \theta_{r}$ are used for angle $\theta_{r}$ and other angles in the rest of the paper. To estimate the robot's attitude, the IMU measurements are used. The angular rate measurements of the IMU are denoted as $\boldsymbol{\Omega}=\left[\begin{array}{lll}\omega_{x} & \omega_{y} & \omega_{z}\end{array}\right]^{T}$ in the IMU frame. The kinematic equations for the IMU motion are written as follows [24]:

$$
\begin{aligned}
& \dot{\phi}_{r}=\omega_{x}+\omega_{y} \mathrm{~s}_{\phi_{r}} \tan \varphi_{r}+\omega_{z} \mathrm{c}_{\phi_{r}} \tan \varphi_{r} \\
& \dot{\varphi}_{r}=\omega_{y} \mathrm{c}_{\phi_{r}}+\omega_{z} \mathrm{~s}_{\phi_{r}} \\
& \dot{\theta}_{r}=\frac{\mathrm{s}_{\phi_{r}}}{\mathrm{c}_{\varphi_{r}}} \omega_{y}+\frac{\mathrm{c}_{\phi_{r}}}{\mathrm{c}_{\varphi_{r}}} \omega_{z} .
\end{aligned}
$$

The discrete-time representation for the system model is used for presentation clarity. The state variable is defined as

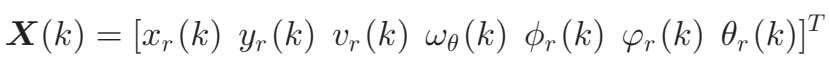

at the $k$ th step. The kinematic motions given in (1) and (2) are linearized and the dynamic model is obtained as

$$
\boldsymbol{X}(k+1)=\boldsymbol{A} \boldsymbol{X}(k)+\boldsymbol{B} \boldsymbol{u}(k)+\boldsymbol{w}(k)
$$

where matrices $\boldsymbol{A}$ and $\boldsymbol{B}$ are given in (4), shown at the bottom of the page, $T$ is the sampling time, $\boldsymbol{u}(k)=\left[\begin{array}{lll}a_{x}(k) & a_{y}(k)\end{array}\right]^{T}$ is the acceleration of the robot motion along the $X-$ and $Y-$ directions, $\boldsymbol{w}(k) \sim \mathcal{N}(\mathbf{0}, \boldsymbol{Q}(k))$ is the Gaussian process noise with covariance $\boldsymbol{Q}(k)=\operatorname{diag}\left\{\sigma_{x}^{2}(k) \sigma_{y}^{2}(k) \sigma_{v_{r}}^{2}(k) \sigma_{\omega_{\theta}}^{2}(k) \sigma_{\phi_{r}}^{2}(k)\right.$ $\left.\sigma_{\varphi_{r}}^{2}(k) \sigma_{\theta_{r}}^{2}(k)\right\}$. Compared with (1), the linear time varying model (3) includes the robot acceleration $\boldsymbol{u}(k)$ to enhance the localization accuracy.

The measurement model of the system is

$$
\boldsymbol{Y}(k)=\boldsymbol{H}(k) \boldsymbol{X}(k)+\boldsymbol{v}(k)
$$

where $\boldsymbol{H}(k)=\left[\begin{array}{ll}\boldsymbol{I}_{4} & \mathbf{0}_{4 \times 3}\end{array}\right], \boldsymbol{I}_{n}$ is an $n \times n$ identify matrix, $\boldsymbol{v}(k)$ are the observation noises, which are assumed to be zero mean Gaussian white signals with covariance matrix $\boldsymbol{R}(k)$, namely, $\boldsymbol{v}(k) \sim \mathcal{N}(\mathbf{0}, \boldsymbol{R}(k))$. The measurements are obtained as follows: positioning information $\left(x_{r}, y_{r}\right)$ are obtained from the RTK GPS, the robot's linear velocity $v_{r}$, and the yaw angular velocity $\omega_{\theta}$ are obtained by the lower level robot controller.

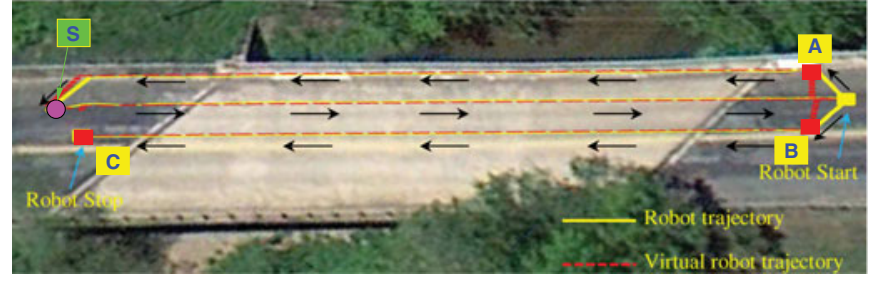

Fig. 7. Schematic of motion planning of the inspection robot on a bridge (the picture shown here is the top view of the Pohatcong Creek Bridge near the township of Bloomsbury, Warren county, NJ, USA.)

The covariance matrix for the measurements are given as $\boldsymbol{R}(k)=\operatorname{diag}\left\{\sigma_{x_{g p s}}^{2}(k) \sigma_{y_{g p s}}^{2}(k) \sigma_{v_{\text {odo }}}^{2}(k) \sigma_{\omega_{\text {odo }}}^{2}(k)\right\}$, which are tuned in the EKF design by using the specification data for the GPS, the IMU, and the robot.

Using the state dynamic model (3) and output relationship (5), an EKF was designed to estimate the robot positioning information $\left(x_{r}, y_{r}\right)$ and attitude heading $\theta_{r}$. Due to the page limit, the detailed description of the EKF design is omitted here and the readers can refer to any similar design such as the one in [24].

\section{B. Path Planning and Robot Motion Control}

The goal of the motion planning and control is to generate the desired trajectory for the robot and then to control the robot to follow the trajectory precisely. The inspected bridge is assumed to be straight and the bridge deck area is assumed to be of a rectangular shape. These assumptions are valid for most bridges. The robot motion planning is indeed a coverage planning problem [31]. A boustrophedon decomposition, also the so-called "ox plowing motion" or trapezoidal decomposition in robotics research, is used [31]. Fig. 7 illustrates the robot motion on a bridge and a brief discussion is presented here to illustrate how to generate the robot motion trajectory. To cover the desired deck area, say the half of the bridge deck surface shown in Fig. 7, three GPS waypoints are first obtained at the rectangle corners such as points $A, B$, and $C$. Using the GPS waypoints of these three corners, the zigzag-shape robot motion trajectories (with interpolated waypoints) are computed by the trapezoidal decomposition algorithm, as the arrows indicate in the figure.

Once the desired trajectory for the robot is determined, a motion control algorithm is then designed for the robot to follow

$$
\boldsymbol{A}=\left[\begin{array}{ccccccc}
1 & 0 & T \mathrm{c}_{\theta_{r}} & 0 & 0 & -T v_{r} \mathrm{~s}_{\theta_{r}} & 0 \\
0 & 1 & T \mathrm{~s}_{\theta_{r}} & 0 & 0 & T v_{r} \mathrm{c}_{\theta_{r}} & 0 \\
0 & 0 & 1 & 0 & 0 & 0 & 0 \\
0 & 0 & 0 & 1 & 0 & 0 & 0 \\
0 & 0 & 0 & 0 & 1+T \dot{\varphi}_{r} \tan \varphi_{r} & \frac{T \dot{\theta}_{r}}{\mathrm{c}_{\varphi_{r}}} & 0 \\
0 & 0 & 0 & 0 & -T \dot{\theta}_{r} \mathrm{c}_{\varphi_{r}} & 1 & 0 \\
0 & 0 & 0 & 0 & \frac{T \dot{\varphi}_{r}}{c_{\varphi_{r}}} & T \dot{\theta}_{r} \tan \varphi_{r} & 1
\end{array}\right]_{\boldsymbol{X}=\boldsymbol{X}(k)} \quad, \quad \boldsymbol{B}=\left[\begin{array}{cc}
\frac{T^{2}}{2} & 0 \\
0 & \frac{T^{2}}{2} \\
0 & 0 \\
0 & 0 \\
0 & 0 \\
0 & 0 \\
0 & 0
\end{array}\right]
$$


the trajectory using an artificial potential field approach. It is considered that a virtual robot is moving along the desired trajectory and generates an attractive force to the inspection mobile robot to follow. Fig. 6(b) illustrates the concept of the virtual robot following design.

The robot velocity vector $\boldsymbol{v}_{r}=\dot{\boldsymbol{q}}_{r} \in \mathbb{R}^{2}$, and the position vector, the velocity vector, and the yaw angle of the virtual robot in frame $\mathcal{I}$ are denoted as $\boldsymbol{q}_{v}=\left[x_{v} y_{v}\right]^{T} \in \mathbb{R}^{2}, \boldsymbol{v}_{v}=\dot{\boldsymbol{q}}_{v}$, and $\theta_{v}$, respectively. The relative position vector and the relative angle from the mobile robot to the virtual robot in frame $\mathcal{I}$ are defined as $\boldsymbol{q}_{r v}=\left[\begin{array}{ll}x_{r v} & y_{r v}\end{array}\right]^{T}$ and $\varphi_{r v}$, respectively; see Fig. 6(b). The value of $\boldsymbol{q}_{r v}$ is calculated as

$$
x_{r v}=x_{v}-x_{r}, \text { and } y_{r v}=y_{v}-y_{r} .
$$

Similarly, the relative velocity $\boldsymbol{v}_{r v}=\left[\begin{array}{ll}\dot{x}_{r v} & \dot{y}_{r v}\end{array}\right]^{T}$ between the actual and virtual robots are computed as

$$
\dot{x}_{r v}=v_{v} \mathrm{c}_{\theta_{v}}-v_{r} \mathrm{c}_{\theta_{r}}, \dot{y}_{r v}=v_{v} \mathrm{~s}_{\theta_{v}}-v_{r} \mathrm{~s}_{\theta_{r}}
$$

where $v_{v}$ is the magnitude of $\boldsymbol{v}_{v}$.

The goal of the tracking controller is then to regulate $\left\|\boldsymbol{q}_{r v}\right\|$ to zero as fast as possible, that is, $\boldsymbol{q}_{r} \rightarrow \boldsymbol{q}_{v}$. To design such a controller, the potential field approach [32] is used and an attractive potential function is defined as follows:

$$
U_{a}=\frac{1}{2} \alpha\left\|\boldsymbol{q}_{r v}\right\|^{2}=\frac{1}{2} \alpha \boldsymbol{q}_{r v}^{T} \boldsymbol{q}_{r v}
$$

where $\alpha>0$ is a constant. To track the virtual robot, the velocity controller of the robot is designed as

$$
\boldsymbol{v}_{r}^{d}=\nabla_{\boldsymbol{q}_{r v}} U_{a}=\alpha \boldsymbol{q}_{r v}
$$

where operator $\nabla_{\boldsymbol{x}} U$ represents the gradient of scalar $U$ along the direction of vector $\boldsymbol{x}$. For tracking a moving target (i.e., the virtual robot), (8) is modified and the desired velocity $\boldsymbol{v}_{r}^{d}$ of the mobile robot is computed as

$$
\boldsymbol{v}_{r}^{d}=\boldsymbol{v}_{v}+\alpha \boldsymbol{q}_{r v} .
$$

From (9), the desired velocity magnitude for the robot is calculated as

$$
\left\|\boldsymbol{v}_{r}^{d}\right\|=\sqrt{v_{v}^{2}+2 \alpha v_{v}\left\|\boldsymbol{q}_{r v}\right\| \mathrm{c}_{\theta_{v}-\varphi_{r v}}+\alpha^{2}\left\|\boldsymbol{q}_{r v}\right\|^{2}} .
$$

Note that for tracking the virtual robot, $\left|\theta_{v}-\varphi_{r v}\right| \leq \frac{\pi}{2}$, namely, the virtual robot's velocity must be within the forward direction relative to the robot; see Fig. $6(\mathrm{~b})$. Thus, $c_{\theta_{v}-\varphi_{r v}} \geq 0$ and from (10)

$$
\left\|\boldsymbol{v}_{r}^{d}\right\| \geq v_{v}
$$

is obtained.

It is also desirable to have the equal projected velocities of the virtual and actual robots along the direction perpendicular to the line $l_{1} l_{2}$ connecting their centers, as shown in Fig. 6(b). Therefore, the following relationship is obtained:

$$
v_{r} \mathrm{~s}_{\theta_{r}-\varphi_{r v}}=v_{v} \mathrm{~s}_{\theta_{v}-\varphi_{r v}} .
$$

From the previous equation, the yaw angle controller for the mobile robot is obtained as

$$
\theta_{r}^{d}=\varphi_{r v}+\sin ^{-1}\left(\frac{v_{v} \mathrm{~s}_{\theta_{v}-\varphi_{r v}}}{v_{r}}\right) .
$$

Since the lower level robot control is implemented through fast real-time systems and each wheel is driven by a powerful motor, we assume that the robot velocity $\boldsymbol{v}_{r}$ quickly reaches to its desired value $\boldsymbol{v}_{r}^{d}$. Then from (11), ratio $v_{v} / v_{r} \leq 1$ is obtained. Moreover, when the value of $v_{r}$ is near zero, a threshold value $v_{r \text { min }}$ is used for the calculation given in (12) in practice. Therefore, the calculation of $\theta_{r}^{d}$ in (12) is always obtained.

Although the previously discussed motion planning algorithm generates the desired trajectory, it is still needed to generate the virtual robot's velocity profile $\boldsymbol{v}_{v}$ in order to use (10). The virtual robot's velocity profile is determined and generated by the NDE inspection need. For example, in the field deployment, the robot stops at each $0.6 \mathrm{~m}$ to take one NDE sensor measurement, especially for the impact echo and electrical resistivity sensors. The value of $v_{v}$ is then designed to ramp up at a fast speed, then slow down once the robot reaches around the targeted traveling distance. Therefore, using the velocity and yaw angle controllers in (10) and (12), the robot follows the motion of the virtual robot along the predefined path.

At the each end of the planned zigzag trajectories, the robotic system has to turn around about $180^{\circ}$ to start the next scan (see Fig. 7). Since the footprint of the robotic system is large ( $2 \mathrm{~m} \times$ $2 \mathrm{~m}$ ), a special safe turn at these end locations is designed to avoid interference with curbs and bridge structures. The omnidirectional motion algorithm is used to allow the robot to move to the predefined safe locations (i.e., point $S$ in Fig. 7), while still keeping its current heading orientation. The motion control of the robot is similar to the above discussed approach and the detailed design is omitted here.

\section{EXPERIMENTS AND FIELD DEPLOYMENT}

In this section, the navigation testing experiments of the robotic system on one of the Rutgers campuses are presented. This is followed by the field deployment results.

\section{A. Navigation Experiments}

The robot navigation system was extensively tested on Rutgers' Busch campus before it was deployed on bridge decks. Fig. 8 illustrates one example of the comparison of the results of the navigation systems based on the EKF design discussed in Section IV-A. It is clearly shown that the EKF-based navigation system overperforms the localization results based on the wheel odometry. It can be noted that when the robot is running near buildings and trees, the GPS data are unstable and not reliable. The top two subfigures in Fig. 8 illustrate the comparison results of the EKF-based localization with those given by GPS signal only when there are trees and buildings nearby along the trajectory. It can be clearly observed that the trajectories based on the GPS data are noisy and distorted. With the EKF-based sensor fusion, much more accurate localization information is achieved. 


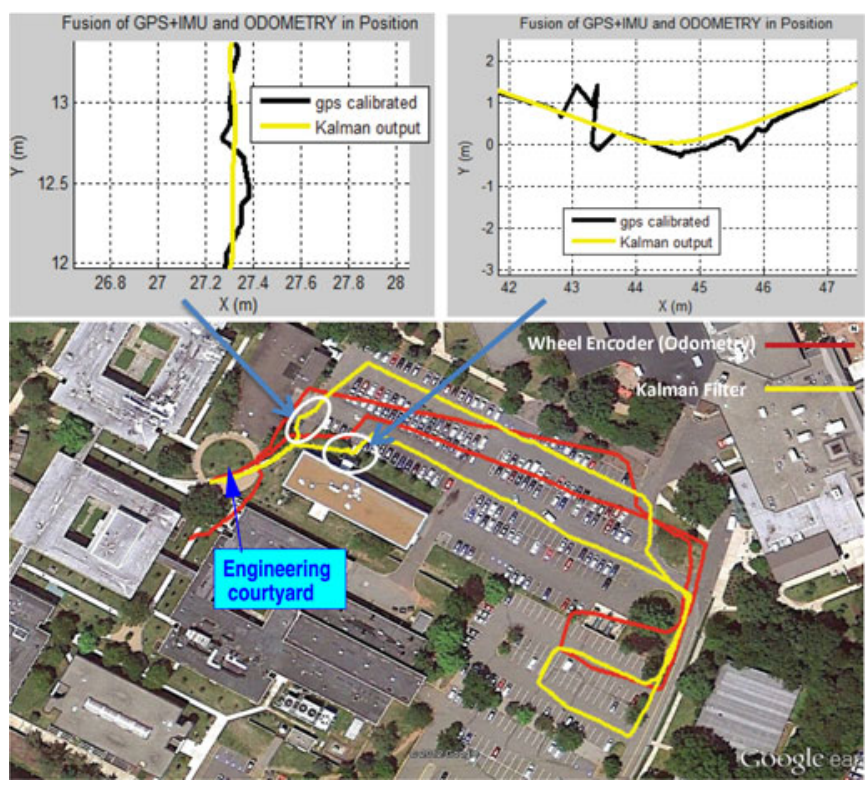

Fig. 8. Comparison of the navigation results by the EKF-based GPS/IMU/ odometry fusion and the odometry-based localization on Rutgers Busch campus.

To further demonstrate the localization and navigation performance, Fig. 9 shows the comparison results among the EKFbased sensor fusion design, the wheel odometry, and the GPS measurements when the GPS signals are not reliable due to the nearby trees along the trajectory. From Fig. 9(a), it is clearly seen that the GPS/IMU/wheel odometry fusion scheme provides similar results to the RTK GPS localization, except at the locations where some trees are nearby. At these locations, the RTK GPS signals are not stable and the errors produced by the GPS data are more than $2 \mathrm{~m}$ although its accuracy specification is within $2-5 \mathrm{~cm}$. The fused GPS/IMU/wheel odometry certainly provides the best localization among all the three localization approaches. It can be also noted that although the wheel odometry cannot produce accurate and reliable localization information over a long period, it does help recover the localization information when the GPS signals are unreliable or even unavailable for a short period of time.

\section{B. Field Deployment}

The NDE sensor arrays and probes were tested and their performance were validated on an actual bridge during the summer and fall of 2012. The Pohatcong Creek Bridge near the township of Bloomsbury, Warren County, NJ, USA, was chosen as the field deployment site. The bridge was built in 1970, and it has a bare concrete deck in a fair condition. The size of the Pohatcong Creek Bridge is about 160 feet $(49 \mathrm{~m})$ long and about 40 feet $(12 \mathrm{~m})$ wide. The top view of the bridge deck from Google Earth and the path during a robot maneuvering test are shown in Fig. 7. Three robotic scans were planned, as shown in the figure.

The testing results for the robot localization and navigation control are shown in Fig. 10. The virtual robot-based motion control design generated satisfactory trajectory-following results. For most of the time of the test run, the tracking errors were within $5-15 \mathrm{~cm}$ among the three scans of the bridge deck.

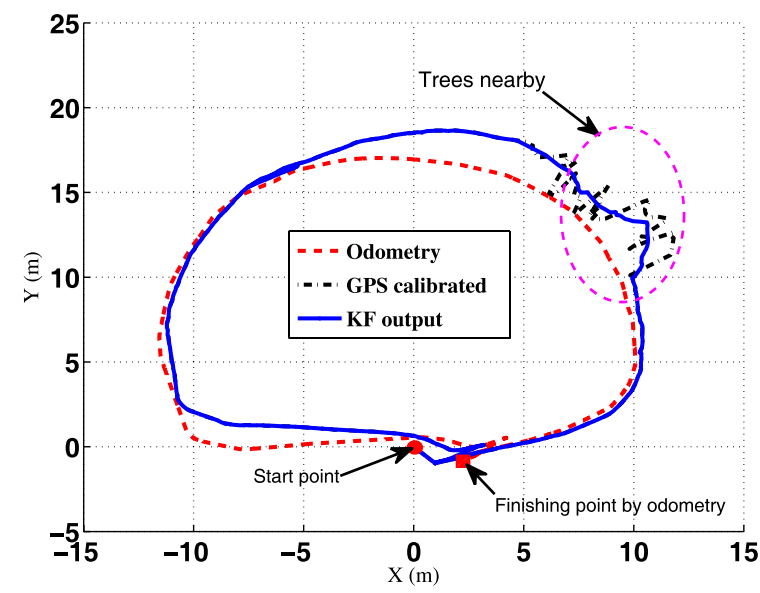

(a)

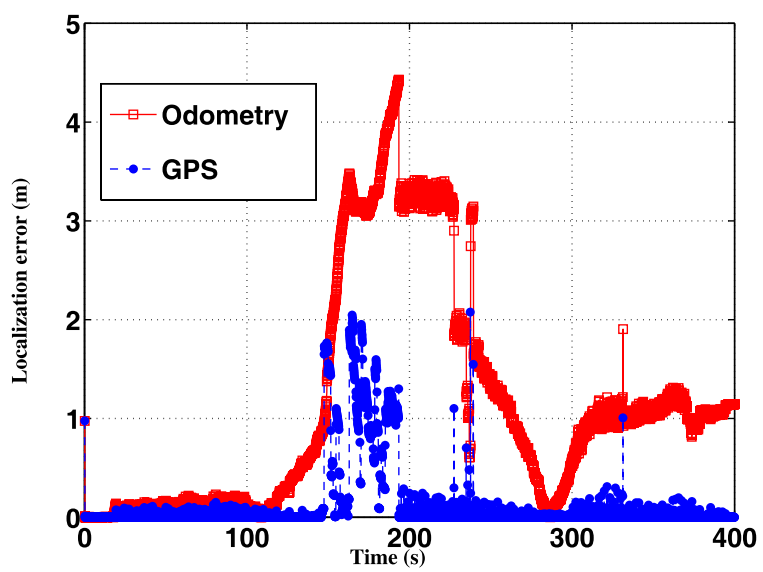

(b)

Fig. 9. Localization comparisons among the GPS/IMU/wheel odometry fusion, the GPS/odometry fusion, and the GPS/IMU fusion when trees are nearby the trajectory. (a) $X Y$ trajectory. (b) Localization errors.

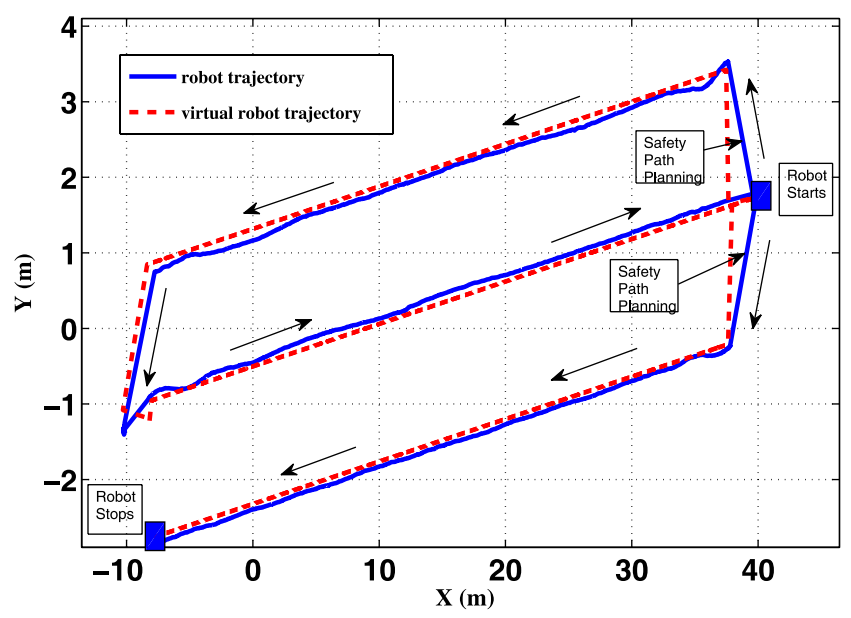

Fig. 10. Localization and navigation results on the Pohatcong Creek Bridge.

The high-accuracy navigation scheme enabled the robotic system to simultaneously deploy the NDE sensors and collect data. The NDE scanning experiments and condition evaluation were conducted on the bridge deck surface. The preliminary tests demonstrated that the throughput performance of the robotic 
NDE systems was around 3-4 times faster than that of the current manually conducted NDE scanning tests. A comprehensive, quantitative comparison of the robotic NDE systems with the current NDE technologies on bridge decks are under development and will be reported in future publications.

\section{CONCLUSION AND FUture WORK}

The development and demonstration of a mechatronic systems design for an autonomous robotic system were presented for high-efficiency bridge deck inspection and evaluation. The main objective of the autonomous robotic NDE system is to increase the inspection efficiency, accuracy, and reduce the risk to bridge inspectors. The developed autonomous inspection system was built on a holonomic mobile robot platform and integrated with multiple NDE technologies such as GPR, impact echo, and electrical resistivity. In this paper, the mechatronic design to integrate the NDE sensors with the mobile robot platform and the development of the robotic navigation system were mainly presented for enabling high-performance bridge deck inspection. The high-accuracy robot localization scheme was built on the EKF-based fusion of the RTK GPS, IMU, and wheel odometry measurements, while the robot motion control was designed through a concept of virtual robot following. The robotic system performance was validated through extensive experimental testing and field deployment.

The performance enhancement for the robotic localization and navigation control is one ongoing research direction. Extensive robotic NDE experiments, advanced data processing, and field deployments are also among the future development tasks.

\section{ACKNOWLEDGMENT}

The authors would like to thank Prof. K. Dana of Rutgers University for her support in the project development. The authors are also grateful to graduate students and research staff P. Kaur, P. Prasanna, K. Lee, Y. Zhang, M. Ezzy, F. Liu, and P. Wang of Rutgers University for their help during the system and field testing experiments.

\section{REFERENCES}

[1] ASCE. (2009) "2009 Report Card for Americas Infrastructure," American Society of Civil Engineers, Tech. Rep., [Online]. Available: http://www. infrastructurereportcard.org

[2] N. Gucunski, M. Yan, Z. Wang, T. Fang, and A. Maher, "Rapid bridge deck condition assessment using three-dimensional virsulization of impact echo data," J. Infrastruct. Syst., vol. 18, no. 1, pp. 12-24, 2012.

[3] K. P. Chong, N. J. Carino, and G. Washer, "Health monitoring of civil infrastructures," Smart Mater. Struct., vol. 12, pp. 483-493, 2003.

[4] N. Gucunski, F. Romero, S. Kruschwitz, R. Feldmann, A. Abu-Hawash, and M. Dunn, "Multiple complementary nondestructive evaluation technologies for condition assessment of concrete bridge decks," Transp. Res. Rec., vol. 2201, pp. 34-44, 2010.

[5] D. Huston, J. Cui, D. Burns, and D. Hurley, "Concrete bridge deck condition assessment with automated multisensor techniques," Struct. Infrastruct. Eng., vol. 7, no. 7-8, pp. 613-623, 2011.

[6] K. R. Kirschke and S. A. Velinsky, "Hisrogram-based approach for automated pavement-crack sensing," J. Transp. Eng., vol. 118, no. 5, pp. 700710, 1992.

[7] D. Hong, S. A. Velinsky, and K. Yamazaki, "Tethered mobile robot for automating highway maintenance operation," Robot. Comput.-Integ. Manuf., vol. 13, no. 4, pp. 297-307, 1997.
[8] S. A. Velinsky, "Heavy vehicle system for automated pavement crack sealing," Int. J. Veh. Design, vol. 1, no. 1, pp. 114-128, 1993.

[9] S. J. Lorenc, B. E. Handlon, and L. E. Bernold, "Development of a robotic bridge maintenance system," Automat. Constr., vol. 9, pp. 251-258, 2000.

[10] F. Yao, G. Shao, B. Takaue, and A. Tamaki, "Automatic concrete tunnel inspection robot system," Adv. Robot., vol. 17, no. 4, pp. 319-337, 2003.

[11] S. N. Yu, J. H. Jang, and C. S. Han, "Auto inspection system using a mobile robot for detecting concrete cracks in a tunnel," Automat. Constr., vol. 16, pp. 255-261, 2007.

[12] P. C. Tung, Y. R. Hwang, and M. C. Wu, "The development of a mobile manipulator imaging system for bridge crack inspection," Automat Constr., vol. 11, pp. 717-729, 2002.

[13] M. Trkov, F. Liu, J. Yi, and H. Baruh, "Study of concrete drilling for automated non-destructive evaluation and rehabilitation system for bridge decks," in Proc. 2011 SPIE Conf. Nondestr. Charact. Comp. Mat. Civil, Aero. Eng., Civil Infrastruct. Homeland Security V, San Diego, CA, USA, 2011, pp. 798 307-1-798 307-9.

[14] F. Liu, M. Trkov, J. Yi, and N. Gucunski, "Modeling and design of percussive drilling for autonomous robotic bridge decks rehabilitation," in Proc. IEEE Conf. Automat. Sci.Eng., Madison, WI, USA, 2013, pp. 1075-1080.

[15] H. G. Sohn, Y. M. Lim, K. H. Yun, and G. H. Kim, "Monitoring crack changes in concrete structures," Comp.-Aided Civil Infrastruct. Eng., vol. 20, pp. 52-61, 2005.

[16] J. K. Oh, G. Jang, S. Oh, J. H. Lee, B. J. Yi, Y. S. Moon, J. S. Lee, and Y. Choi, "Bridge inspection robot system with machine vision," Automat. Constr., vol. 18, pp. 929-941, 2009.

[17] R. S. Lim, H. M. La, Z. Shan, and W. Sheng, "Developing a crack inspection robot for bridge maintenance," in Proc. IEEE Int. Conf. Robot. Autom., Shanghai, China, 2011, pp. 6288-6293.

[18] S. Moon and L. E. Bernold, "Vision-based interactive path planning for robotic bridge paint removal," J. Comput. Civil Eng., vol. 11, no. 2, pp. 113-120, 1997.

[19] D. Zhu, J. Gao, C. Cho, Y. Wang, and K.-M. Lee, "Wireless mobile sensor network for the system identification of a space frame bridge," IEEE/ASME Trans. Mechatronics, vol. 17, no. 3, pp. 499-507, 2012.

[20] L. Yang, Z. Guo, Y. Li, and C. Li, "Posture measurement and coordinated control of twin hoisting-girder transporters based on hybrid network and RTK-GPS,"IEEE/ASME Trans. Mechatronics, vol. 14, no. 2, pp. 141-150, Apr. 2009.

[21] A. Arsenault, S. A. Velinsky, and T. A. Lasky, "A low-cost sensor array and test platform for automated mowing," IEEE/ASME Trans. Mechatronics, vol. 16, no. 3, pp. 592-597, Jun. 2011.

[22] F. Aghili and A. Salerno, "Driftless 3-D attitude determination and positioning of mobile robots by integration of IMU with two RTK GPSs," IEEE/ASME Trans. Mechatronics, vol. 18, no. 1, pp. 21-31, Feb. 2013.

[23] K. Ohno, T. Tsubouchi, B. Shigematsu, and S. Yuta, "Differential GPS and odometry-based outdoor navigation of a mobile robot," Adv. Robot., vol. 18, no. 6, pp. 611-635, 2004.

[24] J. Yi, H. Wang, J. Zhang, D. Song, S. Jayasuriya, and J. Liu, "Kinematic modeling and analysis of skid-steered mobile robots with applications to low-cost inertial measurement unit-based motion estimation," IEEE Trans. Robot., vol. 25, no. 5, pp. 1087-1097, Oct. 2009.

[25] E. North, J. Georgy, U. Iqbal, M. Tarbochi, and A. Noureldin, "Improved inertial/odometry/GPS positioning of wheeled robots even in GPS-denied environments," Global Navig. Satell. Syst.-Sig., Theory Appl., vol. 11, pp. 257-278, 2012.

[26] D. Hong, S. A. Velinsky, and X. Feng, "Verification of a wheeled mobile robot dynamic model and control ramifications," ASME J. Dyn. Syst., Meas., Control, vol. 121, no. 1, pp. 58-63, 1999.

[27] H. La, R. Lim, B. Basily, N. Gucunski, J. Yi, A. Maher, F. Romero, and H. Parvardeh, "Autonomous robotic system for high-efficiency nondestructive bridge deck inspection and evaluation," in Proc. IEEE Conf. Automat. Sci. Eng., Madison, WI, USA, 2013, pp. 1065-1070.

[28] [Online]. Available: http://www.ros.org

[29] Z. W. Wang, M. Zhou, G. G. Slabaugh, J. Zhai, and T. Fang, "Automatic detection of bridge deck condition from ground penetrating radar images," IEEE Trans. Automat. Sci. Eng., vol. 8, no. 3, pp. 633-640, Jul. 2011.

[30] M. Scott, A. Rezaizadeh, A. Delahaza, C. G. Santos, M. Moore, B. Graybeal, and G. Washer, "A comparison of nondestructive evaluation methods for bridge deck assessment," NDT \& E Int., vol. 36, pp. 245-255, 2003.

[31] S. LaValle, Planning Algorithms. New York, NY, USA: Cambridge Univ. Press, 2006, [Online]. Available: http://planning.cs.uiuc.edu/

[32] S. S. Ge and Y. J. Cui, "New potential functions for mobile robot path planing," IEEE Trans. Robot. Automat., vol. 16, no. 5, pp. 615-620, Oct. 2000. 


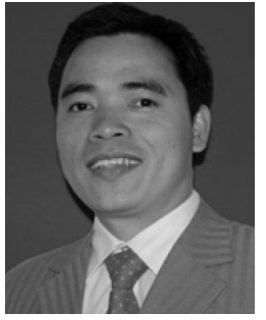

Hung M. La received the B.S. and M.S. degrees in electrical engineering from Thai Nguyen University of Technology, Thai Nguyen, Vietnam, in 2001 and 2003 , respectively, and the Ph.D. degree in electrical engineering from Oklahoma State University, Stillwater, OK, USA, in 2011.

He is currently a Research Associate with the Center for Advanced Infrastructure and Transportation (CAIT), Rutgers University, Piscataway, NJ, USA. He was a Lecturer in the Department of Electrical Engineering, Thai Nguyen University of Technology, from 2001 to 2007. He has been actively involved in research projects with the Federal Highway Administration (FHWA), National Institute of Standards and Technology (NIST), U.S. Department of Transportation (DOT), Department of Defense (DOD), and National Science Foundation (NSF). He is the author of more than 25 papers published in major journals, book chapters and international conference proceedings. His research interests include mobile robotic systems, mobile sensor networks, cooperative control, learning, and sensing, and intelligent transportation systems.

Dr. La received two best paper awards at the 2009 and 2010 Conferences on Theoretical and Applied Computer Science, and one best paper presentation at the network control session of the 2009 American Control Conference.

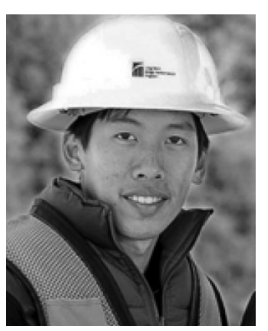

Ronny Salim Lim received the B.S. degree from Pelita Harapan University, Tangerang, Indonesia, in 2008, and the M.S. degree in electrical and computer engineering from Oklahoma State University, Stillwater, OK, USA, in 2011.

$\mathrm{He}$ is currently an Application Developer with the Center for Advanced Infrastructure and Transportation, Rutgers University, Piscataway, NJ, USA His research interests include robotics and automation for civil infrastructure, intelligent transportation systems, and multiagent robotic control

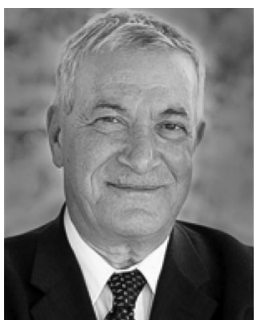

Basily B. Basily received the B.S. and M.S. degrees from Cairo University, Cairo, Egypt, in 1969 an 1972, respectively, and the Ph.D. degree from the University of Astoon, Birmingham, U.K., in 1977, all in mechanical engineering.

He is currently a Research Professor in the Department of Industrial and Systems Engineering, Rutgers University, Piscataway, NJ, USA. He is also a Professor in the Department of Mechanical Design and Production, Cairo University, Giza, Egypt. He is the author of nine international patents and 32 publications in his areas of interest. His research has been funded by the U.S. Navy, National Science Foundation, Department of Defense, Department of Transportation, Honda Corporation, and other industrial companies. His research interests include experimental stress analysis, metal forming, and machine design.

Dr. Basily is a member of the American Society of Engineering Education He received the 2011 Thomas Alva Edison Patent Award from the New Jersey Commission on Science and Technology for his design of a novel folding process and associated machine that manufactures three-dimensional folded core sheet structures.

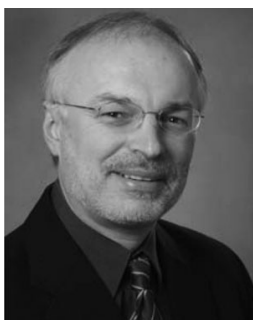

Nenad Gucunski received the B.S. degree in civil engineering from the University of Zagreb, Croatia, and the M.S. degree and the Ph.D. degree in civil engineering from the University of Michigan, Ann Arbor, MI, USA

$\mathrm{He}$ is currently a Professor and Chairman of the Department of Civil and Environmental Engineering, Rutgers University, Piscataway, NJ, USA. He is also the Director of the Infrastructure Condition Monitoring Program at Rutgers' Center for Advanced Infrastructure and Transportation (CAIT). His research interests include NDT/NDE of transportation infrastructure. He is leading a number of important infrastructure-related research projects. He is the PI of the NIST-TIP (National Institute of Standards and Technology-Technology Innovation Program) ANDERS project, SHRP 2 (Strategic Highway Research Project 2) project on NDE for Bridge Decks, the Lead of the NDE Team for FHWA's Long Term Bridge Performance (LTBP) Program, and the PI on several other projects.

Dr. Gucunski is an active member of a number of societies and is serving as the Chair of the Geophysical Engineering Committee of the American Society of Civil Engineers (ASCE).

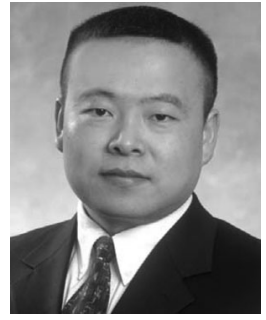

Jingang Yi (S'99-M'02-SM'07) received the B.S degree in electrical engineering from Zhejiang University, Hangzhou, China, in 1993, the M.Eng. degree in precision instruments from Tsinghua University, Beijing, China, in 1996, and the M.A. degree in mathematics and the Ph.D. degree in mechanical engineering from the University of California, Berkeley, CA, USA, in 2001 and 2002, respectively.

$\mathrm{He}$ is currently an Associate Professor of Mechanical Engineering at Rutgers University, Piscataway, NJ, USA. His research interests include autonomous robotic systems, dynamic systems and control, mechatronics, automation science and engineering, with applications to biomedical systems, civil infrastructure, and transportation systems.

Dr. Yi is a member of the American Society of Mechanical Engineers (ASME). He received a 2010 National Science Foundation CAREER Award. He has coauthored papers that have been awarded several best papers at IEEE/ASME AIM, ASME DSCC, IEEE ICRA, etc. He currently serves as an Associate Editor for the IEEE TRANSACTIONS ON AUTOMATION SCIENCE AND ENGINEERING and the IEEE Robotics and Automation Society Conference Editorial Board (since 2008). He also served as a Guest Editor for the IEEE TRANSACTIONS ON AUTOMATION SCIENCE AND ENGINEERING in 2009 and an Associate Editor for the ASME Dynamic Systems and Control Division Conference Editorial Board from 2008 to 2010.

Ali Maher, photograph and biography not available at the time of publication.

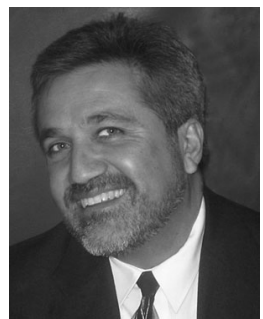

Francisco A. Romero received the B.S. degree in petroleum engineering from the New Mexico Institute of Mining and Technology, Socorro, NM, USA, and the M.S. degree in civil and structural engineering from the University of Kansas, Lawrence, KS, USA.

$\mathrm{He}$ is currently the President of the GPR TEAM Board of Directors, and provides professional GPR/NDE consulting services, training, and business development expertise related to civil/infrastructure NDT applications. Prior to his current position, he was a Senior Research Engineer at the Center for Advanced Infrastructure and Transportation, Rutgers University Piscataway, NJ, USA.

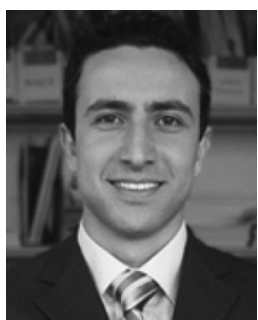

Hooman Parvardeh received the B.S. degree in computer science from Amirkabir University of Technology, Tehran, Iran, in 2008, and the M.S. degree in industrial engineering from Rutgers University, New Brunswick, NJ, USA, in 2011.

$\mathrm{He}$ is currently a Research Engineer at the Center of Advanced Infrastructure and Transportation Rutgers University, Piscataway, NJ, USA. His research interests are nondestructive testing and nondestructive evaluation (NDE) of bridge decks along with bridge data management and bridge manage- 